\title{
DEDUCTIVE SYSTEMS IN COMMUTATIVE PRE-LOGICS
}

\author{
Young Hie $\mathrm{KIm}^{a}$ And Sun SHIN AhN ${ }^{b, *}$
}

\begin{abstract}
The notions of commutative pre-logics and terminal sections are introduced. Characterizations of a commutative prelogic are provided. Properties of deductive systems in pre-logics which are upper semilattices are considered.
\end{abstract}

\section{Introduction}

I. Chajda and R. Halas [1] introduced the concept of a pre-logic which is an algebra weaker than a Hilbert algebra (an algebraic counterpart of intuitionistic logic) but strong enough to have deductive systems. They also studied algebraic properties of pre-logics and of lattices of their deductive systems.

In this paper, we introduced the notion of commutative pre-logics and terminal sections, and give some characterizations of commutative pre-logics in terms of lattices order relations, and terminal sections. We also study properties of deductive systems in pre-logics which are upper semilattices.

\section{Preliminaries}

We recall some definitions and results (see [1]).

Definition 2.1. By a pre-logic, we mean a triple $(X ; \cdot, 1)$ where $X$ is a non-empty set, $\cdot$ is a binary operation on $X$ and $1 \in X$ is a nullary operation such that the following identities hold:

*Corresponding author.

e-mail: yh0314@yonsei.ac.kr (Y.H. Kim), sunshine@dongguk.edu (S. S. Ahn)

Received August 26, 2010. Accepted November 03, 2010.

2000 Mathematics Subject Classification. 06F35, 03G25.

Keywords and phrases. (commutative) pre-logic deductive system(generated by a set) terminal section. 
(P1) $(\forall x \in X)(x \cdot x=1)$,

(P2) $(\forall x \in X)(1 \cdot x=x)$,

(P3) $(\forall x \in X)(x \cdot(y \cdot z)=(x \cdot y) \cdot(x \cdot z))$,

(P4) $(\forall x, y, z \in X)(x \cdot(y \cdot z)=y \cdot(x \cdot z))$.

Lemma 2.2. Let $(X ; \cdot, 1)$ be a pre-logic. Then the following hold:

(a) $(\forall x \in X)(x \cdot 1=1)$;

(b) $(\forall x, y \in X)(x \cdot(y \cdot x)=1)$;

(c) a binary operation $\leq$ on $A$ defined by

$$
(\forall x, y \in X)(x \leq y \text { if and only if } x \cdot y=1)
$$

is a quasiorder on $X$ (i.e., a reflexive and transitive binary relation on $X)$;

(d) $1 \leq x$ for all $x \in X$ implies $x=1$.

REMARK 2.3. The quasiorder $\leq$ of Lemma 2(c) is called the induced quasiorder of a pre-logic $X$.

Lemma 2.4. Let $\leq$ be the induced quasiorder of a pre-logic $X=$ $(X ; \cdot 1)$ and $x, y, z \in X$. If $x \leq y$, then $z \cdot x \leq z \cdot y$ and $y \cdot z \leq x \cdot z$.

Definition 2.5. Let $X=(X ; \cdot, 1)$ be a pre-logic. A non-empty subset $D$ of $X$ is called a deductive system of $X$ if the following conditions hold:

(d1) $1 \in D$;

(d2) if $x \in D$ and $x \cdot y \in D$, then $y \in D$.

LEMMA 2.6. Let $X=(X ; \cdot, 1)$ be a pre-logic and $\leq$ its induced quasi order. The the following hold:

(a) $(\forall x, y \in X)(x \cdot((x \cdot y) \cdot y)=1)$,

(b) $(\forall x, y, z \in X)((y \cdot z) \cdot((x \cdot y) \cdot(x \cdot z))=1)$,

(c) if $D$ is a deductive system of $X$ and $a \in D, a \leq b$, then $b \in D$.

Denote by $\mathcal{D}(X)$ the set of all deductive systems of $X$. It is clear that $\mathcal{D}(X)$ is non-empty.

Lemma 2.7. Let $(X ; *, 1)$ be a pre-logic and $D_{i} \in \mathcal{D}(X)$ for each $i \in I$. Then $D \in \mathcal{D}(X)$ for $D=\cap\left\{D_{i} \mid i \in I\right\}$.

It implies that the set $\mathcal{D}(X)$ forms a closure operator on the power set of $X$. For every subset $A \subseteq X$ there exists the least deductive system on $X$ containing $A$. Denote it by $\langle A\rangle$ and call the deductive systems generated by $A$. If $A=\{a\}$, we will denote $\langle\{a\}\rangle$ briefly by $\langle a\rangle$ and call it the principal deductive system. Hence, if $A, B$ are subsets of $X$, then 
(1) $A \subseteq\langle A\rangle$,

(2) $A \subseteq B$ implies $\langle A\rangle \subseteq\langle B\rangle$,

(3) $\langle\langle A\rangle\rangle=\langle A\rangle$.

If $A=\left\{x_{1}, \cdots, x_{n}\right\}$, then we denoted by $\left\langle x_{1}, \cdots, x_{n}\right\rangle=\left\langle\left\{x_{1}, \cdots, x_{n}\right\}\right\rangle$.

Theorem 2.8. The lattice $\mathcal{D}(X)$ of all deductive systems of a prelogic $X$ is an algebraic lattice whose compact elements are just finitely generated deductive systems. Let $A \subseteq X$. If $A=\emptyset$, then $\langle\emptyset\rangle=\{1\}$; if $A \neq \emptyset$, then

$\langle A\rangle=\left\{a \in X \mid x_{1} \cdot\left(x_{2} \cdot\left(\cdots\left(x_{n} \cdot a\right) \cdots\right)\right)=1\right.$ for some $\left.x_{1}, x_{2}, \cdots x_{n} \in A\right\}$.

Definition $2.9([2])$. A dual $B C K$-algebra is an algebra $(X ; *, 1)$ of type $(2,0)$ satisfying $(\mathrm{P} 1)$, lemma $2.2(\mathrm{a})$, and the following axioms:

(dBCK1) $x * y=y * x=1 \Rightarrow x=y$,

$(\mathrm{dBCK} 2)(x * y) *((y * z) *(x * z))=1$,

(dBCK3) $x *((x * y) * y)=1$.

Proposition $2.10([2])$. Let $(X ; *, 0)$ be a dual $B C K$-algebra and $x, y, z \in X$. Then

(1) $x *(y * z)=y *(x * z)$,

(2) $1 * x=x$.

\section{Commutative pre-logics}

Proposition 3.1. Let $X$ be a pre-logic and let $x, y, z \in X$. If $z \leq x \cdot y$ and $z \leq x$, then $z \leq y$.

Proof. Assume that $z \leq x \cdot y$ and $z \leq x$ for any $x, y, z \in X$. Then $x \cdot y \in\langle z\rangle$ and $x \in\langle z\rangle$. Since $\langle z\rangle$ is a deductive system, it follows from (d2) that $y \in\langle z\rangle$. Hence $z \leq y$.

We now give an equivalent condition of a deductive system.

Theorem 3.2. Let $D$ be a non-empty subset of a pre-logic $X$. Then $D$ is a deductive system of $X$ if and only if for any $x$ and $y$ in $D, x \leq y \cdot z$ implies $z \in D$.

Proof. Let $D$ be a deductive system of $X$ and let $x, y \in D$. If $x \leq y \cdot z$, then $x \cdot(y \cdot z)=1$. Using (d2), we have $y \cdot z \in D$. Using (d2) again, $z \in D$.

Conversely, assume that $x \leq y \cdot z$ implies $z \in D$ for all $x, y \in D$ and $z \in X$. Since $D \neq \emptyset$, we may assume $x \in D$. We note from Lemma 
TABLE $1 . \cdot$-operation

\begin{tabular}{c|ccccc}
\hline$\cdot$ & 1 & $a$ & $b$ & $c$ & $d$ \\
\hline 1 & 1 & $a$ & $b$ & $c$ & $d$ \\
$a$ & 1 & 1 & $b$ & $c$ & $d$ \\
$b$ & 1 & 1 & 1 & $c$ & $d$ \\
$c$ & 1 & 1 & 1 & 1 & $d$ \\
$d$ & 1 & 1 & $b$ & $c$ & 1 \\
\hline
\end{tabular}

2.2(a) that $x \leq x \cdot 1$ so that $1 \in D$ by assumption. Let $x \in D$ and $x \cdot y \in D$. Combining Lemma 2.6(a) and assumption, we get $y \in D$. Hence $D$ is a deductive system of $X$. This completes the proof.

Theorem 3.3. For any deductive system $D$ of a pre-logic $X$ and any $a \in X$, the set $D_{a}:=\{x \in X \mid a \cdot x \in D\}$ is the least deductive system of $X$ containing $D$ and $a$.

Proof. It follows from Lemma 2.2(a) that $a \cdot 1=1$ for any $a \in X$. Hence $1 \in D_{a}$. Using (P1), we have $a \cdot a=1 \in D$ for any $a \in D$ and so $a \in D_{a}$. Let $x \in D_{a}$ and $x \cdot y \in D_{a}$. Then $a \cdot x \in D$ and $a \cdot(x \cdot y) \in D$. Since $a \cdot(x \cdot y)=(a \cdot x) \cdot(a \cdot y) \in D$ and $a \cdot x \in D$, we obtain $a \cdot y \in D$. Hence $y \in D_{a}$. Thus $D_{a}$ is a deductive system. Let $x \in D$. Since $x \cdot(a \cdot x)=1 \in D$ and $D$ is a deductive system of $X$, we get $a \cdot x \in D$. Hence $x \in D_{a}$. Let $H$ be any deductive system of $X$ containing $D$ and $a$. Let $x \in D_{a}$. Then $a \cdot x \in D \subseteq H$. Since $a \in H$ and $H$ is a deductive system of $X$, we have $x \in H$. Therefore $D_{a} \subseteq H$. Thus $D_{a}$ is the least deductive system of $X$ containing $D$ and $a$.

For any $x, y$ in a pre-logic $X$, we define $x \vee y$ as $(y \cdot x) \cdot x$. Under this definition, using Lemma 2.2(a) and (P4), we have

$$
\begin{aligned}
x \cdot(x \vee y) & =x \cdot((y \cdot x) \cdot x) \\
& =(y \cdot x) \cdot(x \cdot x) \\
& =(y \cdot x) \cdot 1=1,
\end{aligned}
$$

i.e., $x \leq x \vee y$. From Lemma 2.6(a), it follows that $y \leq x \vee y$. Hence $x \vee y$ is an upper bound of $x$ and $y$. As easily seen, we have

$\left(c_{1}\right) \quad x \vee x=x$ and $x \vee 1=1 \vee x=1$.

Example 3.4. Let $X:=\{1, a, b, c, d\}$ be a pre-logic with the -operation given by Table 1 . Then $a \vee d=a \neq 1=d \vee a$ and $a$ is the least upper bound of $a$ and $d$. Hence, in general, $x \vee y \neq y \vee x$ and $x \vee y$ may not be the least upper bound of $x$ and $y$. 
TABLE 2. - -operation

\begin{tabular}{c|cccc}
\hline$\cdot$ & 1 & $a$ & $b$ & $c$ \\
\hline 1 & 1 & $a$ & $b$ & $c$ \\
$a$ & 1 & 1 & $b$ & $c$ \\
$b$ & 1 & $a$ & 1 & $c$ \\
$c$ & 1 & $a$ & $b$ & 1 \\
\hline
\end{tabular}

Definition 3.5. A pre-logic $X$ is said to be commutative if it satisfies the following identity

(C) $(y \cdot x) \cdot x=(x \cdot y) \cdot y$, i.e., $x \vee y=y \vee x$

for all $x, y \in X$.

Example 3.6. Let $X:=\{1, a, b, c\}$ be a set with the - -operation given by Table 2 . It is easy to show that $(X ; \cdot, 1)$ is a commutative pre-logic.

Theorem 3.7. If $X$ is a commutative pre-logic $X$, then it is a semilattice with respect to $\vee$.

Proof. Assume that $X$ is a commutative pre-logic. As already seen, $x \vee y$ is an upper bound of $x$ and $y$. We shall show that $x \vee y$ is the least upper bound of $x$ and $y$, To do this, suppose that $x \leq z$ and $y \leq z$. Then $x \cdot z=y \cdot z=1$. Hence by commutative we have (i): $z=1 \cdot z=(x \cdot z) \cdot z=(z \cdot x) \cdot x$ and (ii): $z=1 \cdot z=(y \cdot z) \cdot z=(z \cdot y) \cdot y$. Using (i) and (ii), we have (iii): $z=(z \cdot x) \cdot x=(((z \cdot y) \cdot y) \cdot x) \cdot x$. Set $u=(z \cdot y) \cdot y$. Then $z=(u \cdot x) \cdot x$ follows from (iii). Since $y \leq(z \cdot y) \cdot y=u$, by Lemma 2.4 we have $u \cdot x \leq y \cdot x$. Using Lemma 2.4, we get $(y \cdot x) \cdot x \leq(u \cdot x) \cdot x=z$. Hence we get $x \vee y \leq z$, which shows that $x \vee y$ is the least upper bound of $x$ and $y$. Therefore we have the associative law with respect to $V$. Consequently, $X$ is a semilattice with respect to $V$. The proof is complete.

The converse of Theorem 3.7 is not true as seen in the following example.

Example 3.8. Let $X:=\{1, a, b, c\}$ be a set with the - -operation given by Table 3 . It is easy to check that $X$ is a pre-logic with semilattice with respect to $\vee$. Since $a \vee b=a \neq 1=b \vee a, X$ is not commutative.

Theorem 3.9. Let $X$ be a pre-logic. If $X$ is commutative, then the following properties hold:

$\left(c_{2}\right) y \cdot(x \vee z)=(z \cdot x) \cdot(y \cdot x)$. 
TABLE 3. - -operation

\begin{tabular}{c|cccc}
\hline$\cdot$ & 1 & $a$ & $b$ & $c$ \\
\hline 1 & 1 & $a$ & $b$ & $c$ \\
$a$ & 1 & 1 & $b$ & $c$ \\
$b$ & 1 & 1 & 1 & $c$ \\
$c$ & 1 & 1 & $b$ & 1 \\
\hline
\end{tabular}

$\left(c_{3}\right) x \leq y$ implies $x \vee y=y$.

$\left(c_{4}\right) z \leq x$ and $x \cdot z \leq y \cdot z$ imply $y \leq x$.

$\left(c_{5}\right) y \vee x=x \vee(y \vee x)$, i.e., $(x \cdot y) \cdot y=(((x \cdot y) \cdot y) \cdot x) \cdot x$. for all $x, y \in X$.

Proof. $\left(c_{2}\right)$ Using $(\mathrm{P} 4)$, we have $y \cdot(x \vee z)=y \cdot((z \cdot x) \cdot x)=(z \cdot x) \cdot(y \cdot x)$. $\left(c_{3}\right)$ If $x \leq y$, then $y=1 \cdot y=(x \cdot y) \cdot y=y \vee x$. Hence by the commutativity, $x \leq y$ implies $x \vee y=y$.

$\left(c_{4}\right)$ Assume that $X$ is commutative. If $z \leq x$ and $x \cdot z \leq y \cdot z$, then $z \cdot x=1$ and $(x \cdot z) \cdot(y \cdot z)=1$. Using (P2), (P4) and commutative, we have

$$
\begin{aligned}
y \cdot x & =y \cdot(1 \cdot x) \\
& =y \cdot((z \cdot x) \cdot x) \\
& =y \cdot((x \cdot z) \cdot z) \\
& =(x \cdot z) \cdot(y \cdot z) \\
& =1
\end{aligned}
$$

which implies that $y \leq x$.

$\left(c_{5}\right)$ Let $X$ be a commutative pre-logic and let $x, y \in X$. We recall that $x \leq(x \cdot y) \cdot y$ means $x \leq y \vee x$. Then $\left(c_{3}\right)$ yields $x \vee(y \vee x)=y \vee x$, which is $\left(c_{5}\right)$. This completes the proof.

Proposition 3.10. If $(X ; \cdot, 1)$ is a commutative pre-logic, then for all $x, y \in X, x \cdot y=1$ and $y \cdot x=1 \Rightarrow x=y$.

Proof. Suppose that $x \cdot y=1$ and $y \cdot x=1$ for all $x, y \in X$. Then $x=1 \cdot x=(y \cdot x) \cdot x=(x \cdot y) \cdot y=y$.

Theorem 3.11. If $(X ; \cdot, 1)$ is a commutative pre-logic, then $(X ; \cdot, 1)$ is a dual $B C K$-algebra.

Proof. Proposition 3.10 yields (dBCK1). Now let $x, y, z \in X$. Applying (P4) and (C), we have

$$
(y \cdot z) \cdot(x \cdot z)=x \cdot[(y \cdot z) \cdot z]=x \cdot[(z \cdot y) \cdot y]=(z \cdot y) \cdot(x \cdot y) .
$$


TABLE $4 . \cdot$-operation

\begin{tabular}{c|cccc}
\hline$\cdot$ & 1 & $a$ & $b$ & $c$ \\
\hline 1 & 1 & $a$ & $b$ & $c$ \\
$a$ & 1 & 1 & $a$ & $a$ \\
$b$ & 1 & 1 & 1 & $a$ \\
$c$ & 1 & 1 & $a$ & 1 \\
\hline
\end{tabular}

Hence

$$
(x \cdot y) \cdot[(y \cdot z) \cdot(x \cdot z)]=(x \cdot y) \cdot[(z \cdot y) \cdot(x \cdot y)] .
$$

Since $x \cdot(y \cdot x)=1$ for any $x \in X$, we have $(x \cdot y) \cdot[(y \cdot z) \cdot(x \cdot z)]=1$. Therefore $(\mathrm{dBCK} 2)$ holds. Moreover, by $(\mathrm{P} 1)$ and $(\mathrm{P} 4), x \cdot((x \cdot y) \cdot y)=$ $(x \cdot y) \cdot(x \cdot y)=1$. From this we have (dBCK3). Thus $X$ is a dual $B C K$-algebra.

The converse of Theorem 3.11 is not true as seen in the following example.

Example 3.12. Let $X:=\{1, a, b, c\}$ be a set with the $\cdot$-operation given by Table 4 . It is easy to check that $X$ is a dual $B C K$-algebra. Since $(a \cdot a) \cdot(a \cdot b)=a \neq 1=a \cdot(a \cdot b), X$ is not a pre-logic.

For an element $a$ of a pre-logic $X$, we consider the set

$$
\{x \in X \mid a \leq x\},
$$

denoted by $H(a)$, which is called the terminal section of an element $a$. Since $1, a \in H(a), H(a)$ is not empty. Using this notation, we can characterize a commutative pre-logic.

Theorem 3.13. If a pre-logic $X$ is commutative, then it satisfies the identity:

(c $\left.c_{6}\right) \quad H(a) \cap H(b)=H(a \vee b)$

for all $a, b \in X$.

Proof. Let $X$ be a commutative pre-logic and let $a, b \in X$. If $x \in$ $H(a) \cap H(b)$, then $a \leq x$ and $b \leq x$. Hence $a \vee b \leq x$, which implies that $x \in H(a \vee b)$. Hence $H(a) \cap \bar{H}(b) \subseteq H(a \vee b)$. Now if $x \in H(a \vee b)$, then $a \vee b \leq x$. Since $a \vee b$ is an upper bound of $a$ and $b$, it follows that $a \leq x$ and $b \leq x$, i.e., $x \in H(a)$ and $x \in H(b)$. Hence $x \in H(a) \cap H(b)$. Therefore $\left(c_{6}\right)$ holds.

Proposition 3.14. Let $X$ be a pre-logic and $x, y \in X$. Then $\langle x \vee y\rangle \subseteq$ $\langle x\rangle \cap\langle y\rangle$ with equality in a commutative pre-logic. 
Proof. The inclusion $\langle x \vee y\rangle \subseteq\langle x\rangle \cap\langle y\rangle$ is trivial. Conversely, let $X$ be a commutative pre-logic and $a \in\langle x\rangle \cap\langle y\rangle$. Then $\langle x\rangle=\{b \in X \mid x \leq b\}$ and $\langle y\rangle=\{b \in X \mid y \leq b\}$, whence $x \vee y \leq a$ giving $a \in\langle x \vee y\rangle$.

Definition 3.15. A deductive system $D$ is said to be maximal if $D \neq X$ and $D \subseteq D_{1} \subseteq X$ implies $D=D_{1}$ or $D_{1}=X$ for $D_{1} \in \mathcal{D}(X)$.

Theorem 3.16. Let $D$ be a maximal deductive system of a pre-logic $X$. Then for any $x, y \in X$, we have $x \cdot y \in D$ or $y \cdot x \in D$.

Proof. Let $x, y \in X$. If $x \in D$, then $x \leq y \cdot x$ implies $y \cdot x \in D$. Similarly, if $y \in D$, then $x \cdot y \in D$, since $y \leq x \cdot y$. Finally assume that $x \notin D$ and $y \notin D$ and $x \cdot y \notin D$. Then $D_{x \cdot y}=\{z \in X \mid(x \cdot y) \cdot z \in D\}$ is a deductive system containing $D$ and $x \cdot y$. Since $D$ is maximal, $D_{x \cdot y}=X$. Hence $(x \cdot y) \cdot(y \cdot x) \in D$, which implies from $(\mathrm{P} 4)$ that $y \cdot((x \cdot y) \cdot x) \in D$. Using (P2) and Lemma 2.2(b) and (P3), we have

$$
\begin{aligned}
y \cdot x & =1 \cdot(y \cdot x) \\
& =(y \cdot(x \cdot y)) \cdot(y \cdot x) \\
& =y \cdot((x \cdot y) \cdot x) \in D .
\end{aligned}
$$

which completes the proof.

\section{References}

[1] I. Chajda and R. Halas, Algebraic properties of pre-logics, Math. Slovaca 52 (2002), 157-175.

[2] K. H. Kim and Y. H. Yon, Dual BCK-algebra and MV-algebra, Sci. Math. Japon. 66(2007), 247-253.

[3] A. Walendiziak, On Commutative BE-algebras, Sci. Math. Japon. on line (e2007), 585-588.

${ }^{a}$ Department of Mathematics

Yonsei University,

Seoul 120-749, Korea

${ }^{b}$ Department of Mathematics Education

Dongguk University,

Seoul 100-715, Korea 